\title{
Nitroxidergic innervation of human cerebral arteries
}

\author{
Otar M. Taktakishvili, MD ${ }^{1,4}$, Li-Hsien Lin, $\mathrm{PhD}^{1}$, Andrew D. Vanderheyden, $\mathbf{M D}^{3,5}$, Marcus \\ B. Nashelsky, $\mathbf{M D}^{3}$, and William T. Talman, $\mathbf{M D}^{1,2}$ \\ ${ }^{1}$ Department of Neurology, Univ. of lowa, lowa City, IA 52242 \\ 2 VAMC, lowa City, IA 52242 \\ 3 Department of Pathology, Univ. of lowa, lowa City, IA 52242 \\ ${ }^{5}$ Pathology Associates/United Clinical Labs, Dubuque, IA
}

\begin{abstract}
A dense network of nerves containing neuronal nitric oxide synthase is present in cerebral vessels from experimental animals. The nerves may regulate cerebrovascular tone, protect the brain from stroke, and contribute to cluster headaches in humans; but studies in humans have shown only modest nitroxidergic innervation of cerebral vessels. We tested the hypothesis that nerve fibers containing neuronal nitric oxide synthase richly innervate human cerebral arteries. We used immunohistochemical techniques at post mortem and found dense neuronal nitric oxide synthase nerve staining in human cerebral vessel walls consistent with participation of nitroxidergic fibers in human physiological and pathophysiological processes.
\end{abstract}

\section{Keywords}

Cerebral artery; headache; human; nitric oxide; parasympathetic; stroke

Parasympathetic nerves to cerebral vessels may be relevant to cluster headache and stroke (Henninger \& Fisher 2007; Kano et al. 1991; Torelli \& Manzoni 2004). Nitric oxide (NO) released from those nerves may participate in regulation of cerebral blood flow (CBF) (Talman \& Nitschke Dragon 1995;Talman \& Nitschke Dragon 2000) and provide tonic vasodilatory influences on cerebral vessels (Boysen et al. 2009). In experimental animals the enzyme neuronal nitric oxide synthase (nNOS) responsible for production of $\mathrm{NO}^{\bullet}$ is found in nerves that arise from the parasympathetic pterygopalatine ganglion and richly innervate cerebral arteries (Nozaki et al. 1993; Toda et al. 1993; Walters et al. 1986; Yoshida et al. 1993). Indeed, nNOS containing nerves essentially disappear after interruption of those ganglionic nerve fibers (Nozaki et al 1993). Selective inhibition of nNOS blocks vasodilatation that would occur with activation of the fibers (Talman \& Nitschke Dragon 2007). Thus, NO` synthesized by nNOS is critical for that neurally-mediated dilatation. Despite the potential relevance of these nerves to human disease, previous studies (Nozaki et al 1993;Tomimoto et al. 1994) have suggested that there is only minimal nitroxidergic (also

Corresponding Author: William T. Talman, MD, Department of Neurology, Carver College of Medicine, University of Iowa, 200 Hawkins Dr., Iowa City, IA 52242. Tel. \# 319-356-8752, FAX \# 319-384-6229, william-talman@uiowa.edu.

${ }^{4}$ Current affiliation - Department of Emergency Medicine, SUNY, Stony Brook, NY 11794

The authors have no conflicts of interest.

Publisher's Disclaimer: This is a PDF file of an unedited manuscript that has been accepted for publication. As a service to our customers we are providing this early version of the manuscript. The manuscript will undergo copyediting, typesetting, and review of the resulting proof before it is published in its final citable form. Please note that during the production process errors may be discovered which could affect the content, and all legal disclaimers that apply to the journal pertain. 
called "nitrergic") innervation of human cerebral vessels. Although the density of innervation does not necessarily correlate with the physiological relevance of that innervation, we hypothesized that human cerebral vessels, like those studied in experimental animals, also contained dense innervation by nitroxidergic nerves and that studying freshly obtained human tissue would enhance our ability to demonstrate nerve fibers containing nNOS. Here, using fresh unfixed tissue, we demonstrate in humans a rich innervation of cerebral arteries by nerves that are immunoreactive for nNOS. We thus demonstrate the anatomical substrate for involvement of $\mathrm{NO}^{*}$ released from those nerves in modulating cerebrovascular tone in humans as it does in experimental animals.

We performed nNOS immunostaining of human internal carotid, anterior cerebral and basilar arteries. Vessels were collected through the University of Iowa Hospitals and Clinics Autopsy Service from two male patients at necropsy approximately $12-16 \mathrm{hrs}$ after each patient's death and were processed, without fixation, for nNOS immunohistochemistry by methods described below. Neither patient had neurologic disease. One (age 62) had presented with acute peritonitis and died suddenly as a result of cardiopulmonary arrest. The other (age 48) died as a result of renal failure. Examining the tissue already collected postmortem did not require prior approval of the Institutional Review Board at the University of Iowa.

Unless otherwise indicated segments (8-10 mm lengths) of the anterior cerebral and basilar arteries were treated as follows at $25^{\circ} \mathrm{C}: 0.3 \%$ triton for 30 minutes; phosphate buffered saline (PBS) for 10 minutes; $0.3 \% \mathrm{H}_{2} \mathrm{O}_{2}$ in PBS for 30 minutes; PBS for 10 minutes; blocking with $10 \%$ donkey serum in PBS for 60 minutes; overnight incubation with sheep anti-nNOS antibody (K205, 1:3000, a gift from Dr. P. C. Emson) (Herbison et al. 1996) at $4^{\circ} \mathrm{C}$; PBS washes 3 times for 5 minutes each; overnight incubation with biotinylated antisheep $\operatorname{IgG}\left(1: 300\right.$, Jackson ImmunoResearch Labs, Pennsylvania, USA) at $4^{\circ} \mathrm{C}$; PBS washes 3 times for 10 minutes each; incubation with horseradish peroxidase (HRP)-conjugated streptavidin (1:500, Jackson ImmunoResearch Labs, Pennsylvania, USA) for 2 hours; PBS washes 3 times for 5 minutes each; incubation with $0.1 \mathrm{M}$ Tris, pH 7.6 for 5 minutes; and incubation with fleshly prepared $0.005 \%$ 3'-3'-diaminobenzidine tetrahydrochloride (DAB), $0.6 \%$ nickel ammonium sulfate and $0.006 \% \mathrm{H}_{2} \mathrm{O}_{2}$ in $0.1 \mathrm{M}$ Tris, $\mathrm{pH} 7.6$ for $1.5-2.5$ minutes. The reaction was stopped with double-distilled water. Specimens were incised longitudinally and mounted with the luminal surface down on Superfrost Plus slides (Fisher Scientific, New Jersey, USA), air dried, and covered with Permount (Fisher Scientific, New Jersey, USA) and a cover slip.

Many nNOS immunoreactive (IR) nerve fibers were observed in the anterior cerebral (Fig. 1A), basilar (Figure 1B), and internal carotid arteries (data not shown). With omission of the primary antibody there was no IR staining (Figure 1C\&D). With antibody, nNOS-IR fibers were seen either as individual fibers or as bundles of various diameters along the walls these arteries. Large fibers ran parallel to the longitudinal axis of the vessels. Thinner fibers formed a dense mesh and tended to run perpendicular to the vessel's longitudinal axis.

This study shows that nitroxidergic nerves innervate human cerebral blood vessels as has been shown in experimental animals (Nozaki et al 1993;Toda et al 1993; Walters et al 1986; Yoshida et al 1993). In contrast to earlier studies ours shows a robust, rather than sparse, innervation by those fibers (Nozaki et al 1993). It is likely that analysis of fixed tissue in earlier studies explains the much less prominent staining reported previously (Nozaki et al 1993;Tomimoto et al 1994) while our use of fresh, unfixed tissue augmented our ability to demonstrate the extent of the nitroxidergic network of fibers. It is unlikely that our study stained non-nitroxidergic fibers in that the nNOS antibody used in this study has been used and validated in numerous other studies and specifically labels nNOS (Lin et al. 
2000). Though the earlier study of nNOS-IR fibers in human tissue (Nozaki et al 1993) did not specify the age of the subjects from which tissue was obtained, we doubt that subject age explains the differences between that study and our own in that we saw no difference in density of staining or staining patterns in our two adult subjects who differed in age by 14 years. We acknowledge that even the current study may underestimate nNOS-IR found in human cerebral vessels due to the limited number of specimens available to us and to our having obtained tissue 12-16 hours postmortem. Immediate capture of tissue may have allowed demonstration of an even more robust network of nNOS fibers in the vessel walls. The limited tissue samples and limited selection of cerebral vessels precluded our quantifying results or making valid comparisons about distribution of nNOS fibers and nNOS content among different vessels. However, the study does show a rich investment of human cerebral vessels with nNOS-containing nerve fibers and suggests that between vascular beds there are differences of nNOS fiber distribution and density that could reflect different origins of nerve fibers.

In demonstrating a dense nitroxidergic innervation in the wall of human cerebral vessels this study provides anatomical support that $\mathrm{NO}^{\circ}$, derived from nNOS in nerve fibers that innervate cerebral arteries, may participate in regulation of cerebrovascular tone in humans and could thus play role in the pathophysiology of cluster headache and stroke. As noted, each condition has been linked to function of the parasympathetic nerves to forebrain vessels, but more study is needed to determine if $\mathrm{NO}^{\circ}$ released from those nerves participates in their pathogenesis.

\section{Acknowledgments}

Support: Department of Veterans Affairs Merit Review (WTT PI), NIH R01-HL59593 (WTT PI) and R01HL088090 (WTT and LHL PIs).

\section{Reference List}

Boysen N, Nitschke Dragon D, Talman WT. Parasympathetic tonic dilatory influences on cerebral vessels. Journal of the Autonomic Nervous System. 2009; 147(1-2):101-104.

Henninger N, Fisher M. Stimulating circle of Willis nerve fibers preserves the diffusion-perfusion mismatch in experimental stroke. Stroke. 2007; 38(10):2779-2786. [PubMed: 17761922]

Herbison AE, Simonian SX, Norris PJ, Emson PC. Relationship of neuronal nitric oxide synthase immunoreactivity to $\mathrm{GnRH}$ neurons in the ovariectomized and intact female rat. Journal of Endocrinology. 1996; 8:73-82.

Kano M, Moskowitz MA, Yokota M. Parasympathetic denervation of rat pial vessels significantly increases infarction volume following middle cerebral artery occlusion. Journal of Cerebral Blood Flow and Metabolism. 1991; 11(4):628-637. [PubMed: 2050751]

Lin LH, Emson PC, Talman WT. Apposition of neuronal elements containing nitric oxide synthase and glutamate in the nucleus tractus solitarii of rat: a confocal microscopic analysis. Neuroscience. 2000; 96(2):341-350. [PubMed: 10683574]

Nozaki K, Moskowitz MA, Maynard KI, Koketsu N, Dawson TM, Bredt DS, Snyder SH. Possible origins and distribution of immunoreactive nitric oxide synthase-containing nerve fibers in cerebral arteries. Journal of Cerebral Blood Flow and Metabolism. 1993; 13:70-79. [PubMed: 7678014]

Talman WT, Nitschke Dragon D. Inhibition of nitric oxide synthesis extends cerebrovascular autoregulation during hypertension. Brain Research. 1995; 672:48-54. [PubMed: 7749753]

Talman WT, Nitschke Dragon D. Parasympathetic nerves influence cerebral blood flow during hypertension in rat. Brain Research. 2000; 873(1):145-148. [PubMed: 10915822]

Talman WT, Nitschke Dragon D. Selective Neuronal Nitric Oxide Synthase Inhibition Attenuates Breakthrough of Autoregulation during Acute Hypertension. Brain Research. 2007; 1139:126-132. [PubMed: 17291465] 
Toda N, Ayajiki K, Yoshida K, Kimura H, Okamura T. Impairment by damage of the pterygopalatine ganglion of nitroxidergic vasodilator nerve function in canine cerebral and retinal arteries. Circulation Research. 1993; 72:206-213. [PubMed: 8417843]

Tomimoto H, Nishimura M, Suenaga T, Nakamura S, Akiguchi I, Wakita H, Kimura J, Mayer B. Distribution of nitric oxide synthase in the human cerebral blood vessels and brain tissues. Journal of Cerebral Blood Flow and Metabolism. 1994; 14(6):930-938. [PubMed: 7523431]

Torelli P, Manzoni GC. Cluster headache: symptomatic treatment. Neurol Sci. 2004; 25(Suppl 3):S119-S122. [PubMed: 15549518]

Walters BB, Gillespie SA, Moskowitz MA. Cerebrovascular projections from the sphenopalatine and otic ganglia to the middle cerebral artery of the cat. Stroke. 1986; 17(3):488-494. [PubMed: 3715948]

Yoshida K, Okamura T, Kimura H, Bredt DS, Snyder SH, Toda N. Nitric oxide synthaseimmunoreactive nerve fibers in dog cerebral and peripheral arteries. Brain Research. 1993; 629:67-72. [PubMed: 7506984] 

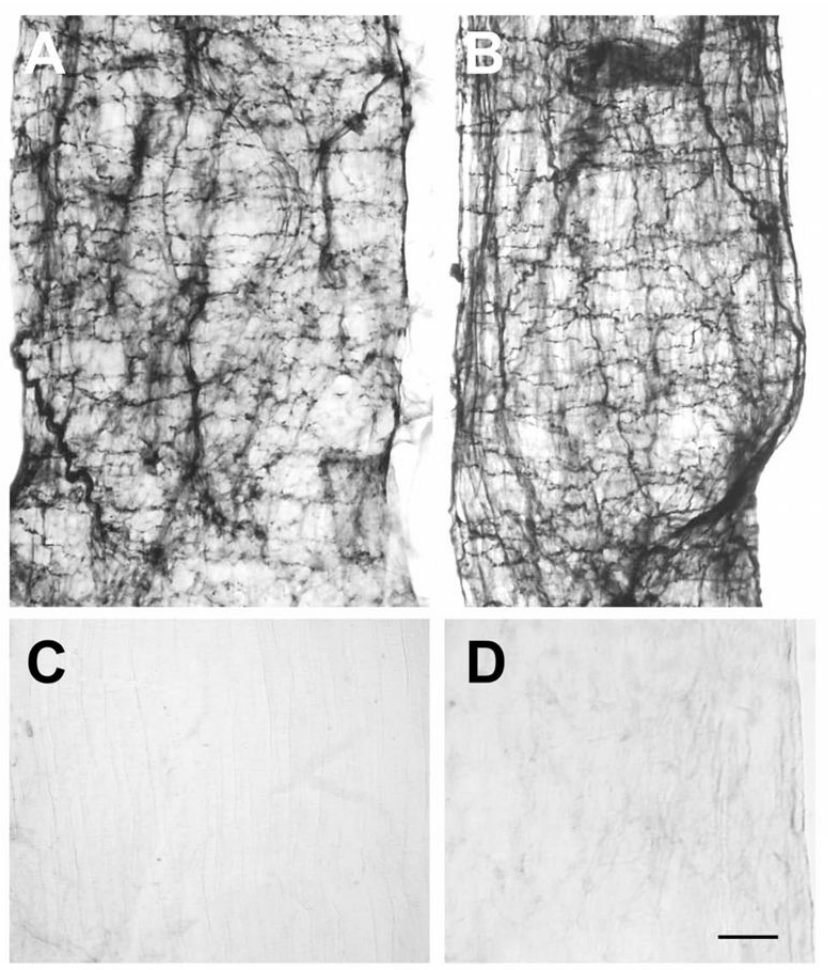

Figure 1.

Photomicrographs show representative immunohistochemical findings. In this subject immunoreactivity (IR) for nNOS is seen in nerve fibers innervating the basilar (A) and anterior cerebral artery (B) but not in the basilar (C) or anterior cerebral artery (D) in the absence of primary antibody. Bar $=100 \mu \mathrm{m}$. 